\title{
Molekulare Diagnostik und Therapie in der Onkologie
}

\author{
Gustavo Baretton $^{\mathrm{a}^{*}} \quad$ Stephan Grabbe $\mathrm{b}^{\mathrm{b}^{*}} \quad$ Ulrich Keilholz ${ }^{\mathrm{c}^{*}} \quad$ Guido Sauter $^{\mathrm{d}^{*}}$ \\ a Institut für Pathologie, Universitätsklinikum "Carl Gustav Carus», TU Dresden, \\ ${ }^{b}$ Hautklinik der Universitätsmedizin, Johannes Gutenberg Universität Mainz, \\ ${ }^{c}$ Medizinische Klinik III und Charité Comprehensive Cancer Center, Berlin, \\ ${ }^{\mathrm{d}}$ Institut für Pathologie, UKE Hamburg-Eppendorf, Deutschland
}

\section{Molekulare Diagnostik in der Onkologie: Status quo und Ausblick}

Individuelle Therapieentscheidungen in der Onkologie basieren zunehmend auf den Ergebnissen molekularbiologischer Diagnoseverfahren, die besonders bei der Targeted Therapy einen wichtigen Beitrag zur Selektion der besonders profitierenden Patienten leisten und damit zur Vermeidung unnötiger Behandlungen und Kosten beitragen können. Bei rasanten technischen Fortschritten, die schon heute die vollständige Sequenzierung des menschlichen Genoms innerhalb einer Woche und in wenigen Jahren vermutlich auch zu relativ günstigen Kosten von etwa $1000 €$ /Analyse ermöglichen, ist die Aussagekraft molekulardiagnostischer Untersuchungen in der täglichen Praxis allerdings durch häufig fehlende Standardisierungen, z.B. bei der Entnahme und Vorbereitung des Tumorgewebes limitiert.

\section{Veränderungen im EGFR-Signaltransduktionsweg}

In zahlreichen soliden Tumoren ist die Signaltransduktion über den Epidermal Growth Factor Receptor (EGFR) pathologisch aktiviert. Eine Verbesserung der Prognose lässt sich bei einem Teil der betroffenen Patienten durch Antikörper der extrazellulären EGFR-Domäne (EGFR-AK) und Inhibitoren der EGFR-Tyrosinkinase (EGFR-TKI) erreichen. Von den EGFR-AK Cetuximab oder Panitumumab profitieren Patienten mit EGFR-positivem kolorektalen Karzinom allerdings nur dann, wenn das Gen für das nachgeschaltete KRAS-Protein nicht mutiert ist, d.h. bei Vorliegen von KRAS-Wildtyp $[1,2]$. Bei der First-Line-Therapie des fort-

\footnotetext{
*Alle Autoren haben zu gleichen Teilen zu diesem Artikel beigetragen.
}

geschrittenen nichtkleinzelligen Lungenkarzinoms (NSCLC) mit den oralen EGFR-TKI Erlotinib oder Gefitinib sind aktivierende Mutationen des EGFR prädiktiv für ein gutes Therapieansprechen [3, 4].

Aktivierende EGFR-Mutationen werden in Europa bei $10-15 \%$ der Patienten mit fortgeschrittenem NSCLC nachgewiesen, wobei etwa 90\% der Mutationen in Exon 19 (Deletionen) und Exon 21 (vor allem Punktmutation L858R) vorliegen [5]. Seltener finden sich Mutationen in Exon 18 (etwa $5 \%)$ und Exon $20(<1 \%)$. Aufgrund der Vielzahl der potenziell betroffenen Exons ist die EGFR-Genmutationsanalyse relativ aufwendig und entsprechend kostenträchtig, sie kann aufgrund der Korrelation von EGFR-Mutationsstatus und Ansprechen auf die First-Line-Therapie des fortgeschrittenen NSCLC (siehe unten) aber einen wichtigen Beitrag zur Selektion der von einer EGFR-TKI-Therapie profitierenden Patienten leisten.

\section{Molekulare Veränderungen beim malignen Melanom}

Bei der Entwicklung von Melanozyten über Nävi zum malignen Melanom in chronisch UV-Licht-geschädigter Haut (CSD) sind Veränderungen im Ras/BRAF/MEK/ERKSignalweg sowie Mutationen und Amplifikationen des für den Stammzellfaktor kodierenden Onkogens c-KIT nachweisbar. Die Häufigkeit der verschiedenen genetischen Anomalien variiert bei unterschiedlichen Subtypen des malignen Melanoms (Tab. 1) [6, 7].

Targets für eine zielgerichtete Therapie sind vor allem die aktivierende BRAF-Mutation V600E in Exon 15 sowie c-KIT-Mutationen. Während bei der Genmutationsanalyse zum Nachweis der in 90\% der BRAF-positiven Melanome nachweisbaren Punktmutation nur ein Exon analysiert werden muss, erfordert der Nachweis von mutiertem c-KIT die Untersuchung der drei Exons 11, 13 und 17.

\begin{tabular}{ll}
\hline KARGER & ( 2011 S. Karger GmbH, Freiburg \\
0378-584X/11/3414-0005 $\$ 38.00 / 0$ \\
$\begin{array}{l}\text { Fax +497614520714 } \\
\text { Information@Karger.de } \\
\text { www.karger.com }\end{array}$ & $\begin{array}{l}\text { Accessible online at: } \\
\text { www.karger.com/onk }\end{array}$
\end{tabular}


Tab. 1. Häufigkeit von Mutationen in Kinase-Signalwegen bei unterschiedlichen Subtypen des malignen Melanoms (modifiziert nach [7])

\begin{tabular}{|c|c|c|c|c|c|}
\hline & Kutan & Akral & Mukosal & Uveal & Nicht spezifiziert \\
\hline BRAF & $40-60$ & $15-20$ & $3-5$ & $<1$ & \\
\hline NRAS & $15-25$ & $10-15$ & $5-15$ & $<1$ & \\
\hline c-KIT (Mutation) & $<2(\mathrm{CSD}: 2-17)$ & $10-20$ & $15-20$ & $<1$ & \\
\hline c-KIT (Amplifikation) & 0-7 (CSD: 6) & $25-30$ & $25-30$ & $<1$ & \\
\hline $\mathrm{GN} \alpha \mathrm{Q}$ & <1 (CSD: 5) & $<1$ & $<1$ & $45-50$ & \\
\hline ERBB4 & & & & & $10-20$ \\
\hline PTEN & & & & & $10-30$ \\
\hline PIK3CA & & & & & $1-2$ \\
\hline AKT1/3 & & & & & $1-2$ \\
\hline
\end{tabular}

\section{Qualitätssicherung in der molekularen Diagnostik}

Die Implementierung molekularpathologischer Tests als Basis individueller Therapieentscheidungen erfordert eine umfassende und regelmäßige Qualitätssicherung mit Standardisierung der relevanten Verfahrensschritte und eine kontinuierliche Überprüfung der Ergebnisse durch Ringversuche. Bei immunhistochemischen Untersuchungen müssen zur Reduktion falsch negativer oder falsch positiver Ergebnisse neben den Analysemethoden und -geräten auch die Entnahme, Lagerung und Vorbereitung des Tumorgewebes standardisiert werden. Bei der Genmutationsanalyse, deren Ergebnisse vor allem bei stark heterogenen Tumoren (z.B. NSCLC, malignes Melanom) durch den Anteil des Tumorgewebes in der Probe beeinflusst werden, ist eine Standardisierung der Gewebedissektion, DNA-Extraktion und Detektion der Genmutation erforderlich; auch die Interpretation der Ergebnisse sollte nach definierten Standards erfolgen.

Um eine breite Anwendung qualitätsgesicherter molekularpathologischer Testverfahren zu ermöglichen, wurden von der Deutschen Gesellschaft für Pathologie und dem Bundesverband Deutscher Pathologen Kriterien für qualitativ hochwertige Tests definiert, die bei der Zertifizierung als Referenzlabor erfüllt sein müssen. Im Rahmen der Qualitätssicherungsinitiative in der Pathologie $\left(\mathrm{QuIP}{ }^{\circledR}\right)$ werden außerdem systematisch wichtige onkologische Biomarker untersucht und deutschlandweit entsprechende Ringversuche durchgeführt.

\section{Ausblick: Tumorprofilierung}

Mittels DNA-Chips kann in frischem Tumorgewebe die Expression von Tausenden von Genen gleichzeitig analysiert werden. Bei Patientinnen mit Mammakarzinom im Stadium I oder II wurde 2002 eine 70-Gensignatur identifiziert, mit der Gruppen mit guter und ungünstiger Prognose unterschieden werden können (Abb. 1) [8]. Basierend auf diesen Daten wurde 2005 der MammaPrint-Test entwickelt, der 2007 von der Food and Drug Administration (FDA) zugelassen wurde und heute wie zahlreiche weitere kommerzielle Gen-Chips

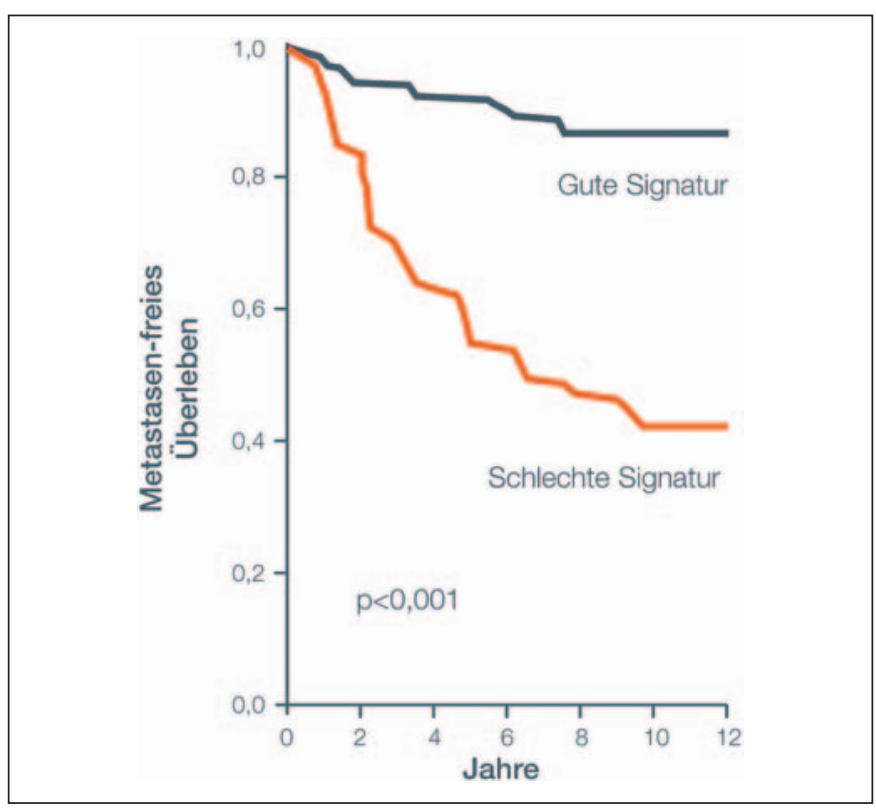

Abb. 1. Metastasenfreies Überleben beim Mammakarzinom im Stadium I oder II (modifiziert nach [8]).

beim Mammakarzinom zur Vorhersage der Prognose und Therapieentscheidung genutzt wird. Weitere DNA-Chips werden beim Mammakarzinom zur Identifikation einer Prädisposition und zur Früherkennung eingesetzt.

\section{Entwicklung zielgerichteter Therapeutika in der Onkologie}

\section{Beispiel Lungenkarzinom}

Mit jährlich etwa 40000 Neuerkrankungen zählt das Lungenkarzinom in Deutschland zu den häufigsten Tumoren. Neben der hohen Inzidenz und der bislang sehr ungünstigen Prognose stellt die histologische und molekularbiologische Heterogenität beim NSCLC besondere Anforderungen an die Diagnostik und Therapie.

Da das Potenzial der Chemotherapeutika beim NSCLC vermutlich weitestgehend ausgeschöpft ist, sind weitere Ver- 


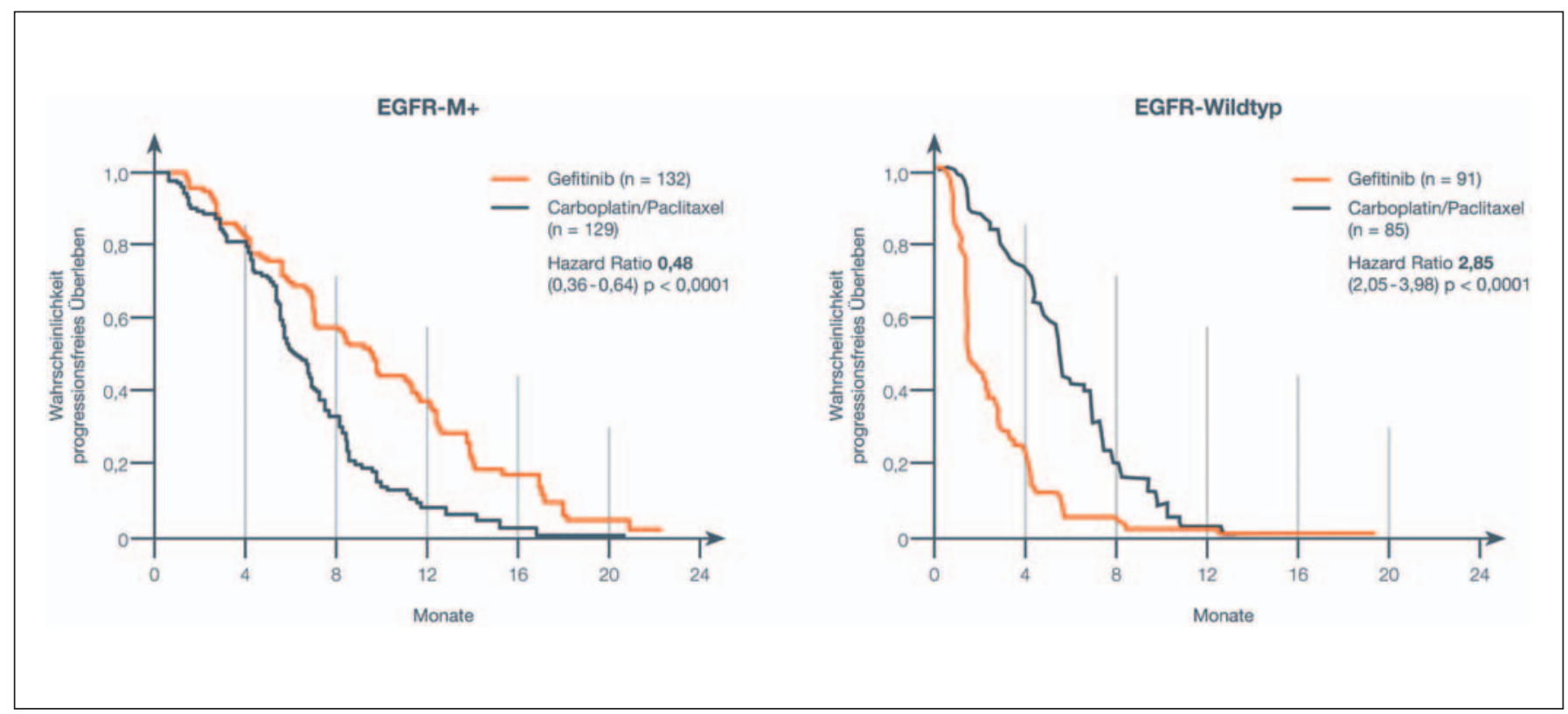

Abb. 2. IPASS-Studie: Progressionsfreies Überleben bei Nachweis aktivierender EGFR-Mutationen und EGFR-Wildtyp (Intention-To-Treat (ITT)-Population) (modifiziert nach [12]).

besserungen der Prognose hier wie auch beim malignen Melanom und zahlreichen anderen soliden Tumoren zukünftig wahrscheinlich nur durch personalisierte Therapieansätze mit Integration von Targeted Drugs und Biologicals zu erwarten. Beim fortgeschrittenen NSCLC wurden als wichtige «onkogene Drivers» bisher vor allem aktivierende Mutationen des EGFR sowie ein EML4-ALK-Fusionsgen identifiziert. Diese Veränderungen wurden allerdings nur in kleinen Patientengruppen (vor allem bei Nichtrauchern) nachgewiesen. Als weitere mögliche Angriffspunkte für eine zielgerichtete Therapie werden zurzeit unter anderem HER2, cMET und PIK3CA untersucht.

\section{Zielstruktur EGFR}

Eine unkontrollierte Aktivierung der über EGFR vermittelten Signalkaskade lässt sich beim NSCLC sowohl durch EGFR-AK als auch durch EGFR-TKI hemmen. Während die beiden EGFR-TKI Erlotinib und Gefitinib bereits zur Therapie des fortgeschrittenen NSCLC zugelassen sind, ist die Datenlage zum Einsatz von EGFR-AK in dieser Indikation noch unzureichend.

Der EGFR-TKI Erlotinib wurde bei chemotherapeutisch vorbehandelten Patienten mit fortgeschrittenem NSCLC zugelassen, nachdem er in der placebokontrollierten Phase-IIIStudie BR.21 das mediane Gesamtüberleben im Vergleich zu einer optimalen Supportivtherapie (BSC) um 2 Monate verlängerte (6,7 vs. 4,7 Monate) und das Mortalitätsrisiko statistisch signifikant um 30\% reduzierte (Hazard Ratio (HR) 0,70; $\mathrm{p}<0,001)$. Bei einer Verringerung des Progressionsrisikos um $39 \%$ (HR 0,61; $p<0,001$ ) [9] verlängerte Erlotinib zugleich die Zeit bis zur Verschlechterung der krankheitsspezifischen Symptome [10].
Erste Hinweise auf Besonderheiten in der Tumorbiologie des NSCLC gaben die Daten von Lynch et al. [11], die bei 8 von 9 Patienten mit ungewöhnlich langen Ansprechzeiten auf Gefitinib aktivierende Mutationen im EGFR-Gen (Exon 18, 19 und 21) fanden. Alle Langzeit-Responder waren Nichtraucher oder Exraucher und an einem Adenokarzinom oder bronchioloalveolären Karzinom (BAC) erkrankt. Indirekt bestätigt wurde diese Beobachtung unter anderem durch die Ergebnisse der Phase-III-Studie IPASS, in der bei NSCLC-Patienten mit klinischer Anreicherung von aktivierenden EGFR-Mutationen (Asiaten, Adenokarzinom, Nichtraucher oder geringer Nikotinkonsum in der Vergangenheit) in der First-Line-Therapie Gefitinib mit einer Standardchemotherapie (Carboplatin/Paclitaxel) verglichen wurde. Der EGFR-TKI reduzierte das Progressionsrisiko im Gesamtkollektiv um 26\% (HR 0,74; p < 0,001), wobei sich allerdings die Kaplan-Maier-Kurven nach etwa 5 Monaten überschnitten [4]. Ursächlich für die ungewöhnlichen Verläufe des progressionsfreien Überlebens (PFS) könnte die Heterogentität des Studienkollektivs sein, das aufgrund des Studiendesigns nicht nur Patienten mit aktivierenden EGFR-Mutationen (EGFR-M+), sondern auch solche mit EGFR-Wildtyp umfasste.

In Abhängigkeit vom EGFR-Mutationsstatus durchgeführte Subanalysen zeigten, dass Patienten mit EGFR-M+ bei einem im Vergleich zu Patienten mit EGFR-Wildtyp insgesamt längeren PFS einen statistisch signifikanten Vorteil von der EGFR-Hemmung hatten (HR 0,48; $\mathrm{p}<0,0001$ ). Bei Patienten mit nicht mutiertem EGFR war Gefitinib der Chemotherapie dagegen signifikant unterlegen (HR 2,85; $<<0,0001$ ) (Abb. 2) [12]. 


\section{Zielstruktur EML4-ALK}

Bei etwa $5 \%$ der Patienten mit fortgeschrittenem NSCLC lässt sich ein EML4-ALK-Fusionsprotein nachweisen, das verschiedene für die Tumorzellproliferation wichtige Signaltransduktionswege unkontrolliert aktiviert. Wie aktivierende EGFR-Mutationen werden auch EML4-ALK-Fusionsgene überwiegend bei Nichtrauchern und Patienten mit Adenokarzinom gefunden.

Der selektive, oral wirksame ALK-Inhibitor Crizotinib zeichnete sich bereits in einer ersten Studie bei überwiegend stark vorbehandelten Patienten mit ALK-positivem NSCLC durch eine bemerkenswert hohe Aktivität aus. Eine bei der American Society of Clinical Oncology (ASCO)-Jahrestagung 2010 präsentierte Interims-Analyse einer Phase-I/IIStudie mit 82 Patienten zeigte eine Rate der bestätigten partiellen (PR) oder kompletten Remissionen (CR) von 57\%, bei weiteren $6 \%$ der Patienten war die Response zum Zeitpunkt der Datenanalyse noch nicht bestätigt. Die Krankheitskontrollrate betrug $87 \%$, der Anteil der Patienten ohne Krankheitsprogression nach 6 Monaten wurde zum damaligen Zeitpunkt auf $72 \%$ geschätzt. Bei insgesamt guter Verträglichkeit wurden als häufigste unerwünschte Ereignisse leichte bis moderate Übelkeit und Erbrechen berichtet [13].

\section{Molekulare Therapieansätze beim metastasierten Melanom}

Wie das NSCLC zeichnet sich auch das Melanom durch eine ausgeprägte molekulare Heterogenität aus, wobei für verschiedene Melanomtypen eine Assoziation mit bestimmten genetischen Signaturen gezeigt wurde [14]. Als mögliche Targets für eine zielgerichtete Therapie eignen sich nach den bisher vorliegenden Daten vor allem Mutationen im Ras/ BRAF/MEK/ERK-Signalweg sowie aktivierende c-KITMutationen. $\mathrm{Zu}$ den molekularen Targets für eine Immuntherapie zählen MHC-bindende Peptide und Proteine, Melanom- bzw. Tumor-spezifische Oberflächenantigene, kostimulierende Moleküle wie CD40 und koinhibitorische Moleküle, beispielsweise CTLA-4, PD-1, PD-L1 und PD-L2.

\section{Zielstruktur Ras/BRAF/MEK/ERK-Signaltransduktionsweg} BRAF-Inhibitoren: Bei bis zu $60 \%$ der kutanen Melanome ist eine aktivierende BRAF-Mutation nachweisbar, wobei in 90\% dieser Fälle die V600E-Punktmutation vorliegt. Eine dadurch induzierte konstitutive Aktivierung der RAF/MEK/ ERK-Signalkaskade lässt sich durch Inhibitoren der BRAFKinase, wie PLX4032 oder GSK2118436, sowie MEK-Inhibitoren, wie GSK1120212 oder AZD6244, hemmen (Abb. 3).

Der niedermolekulare, oral wirksame, selektive BRAFInhibitor PLX4032 zeigte in einer Phase-I/II-Studie, an der in der Dosis-Eskalationsphase 55 Patienten, darunter 49 mit metastasiertem Melanom, teilnahmen, eine bemerkenswert hohe Aktivität. In der Extensionsphase, in der ausschließlich Melanom-Patienten mit der BRAF-Mutation V600E $(\mathrm{n}=32)$

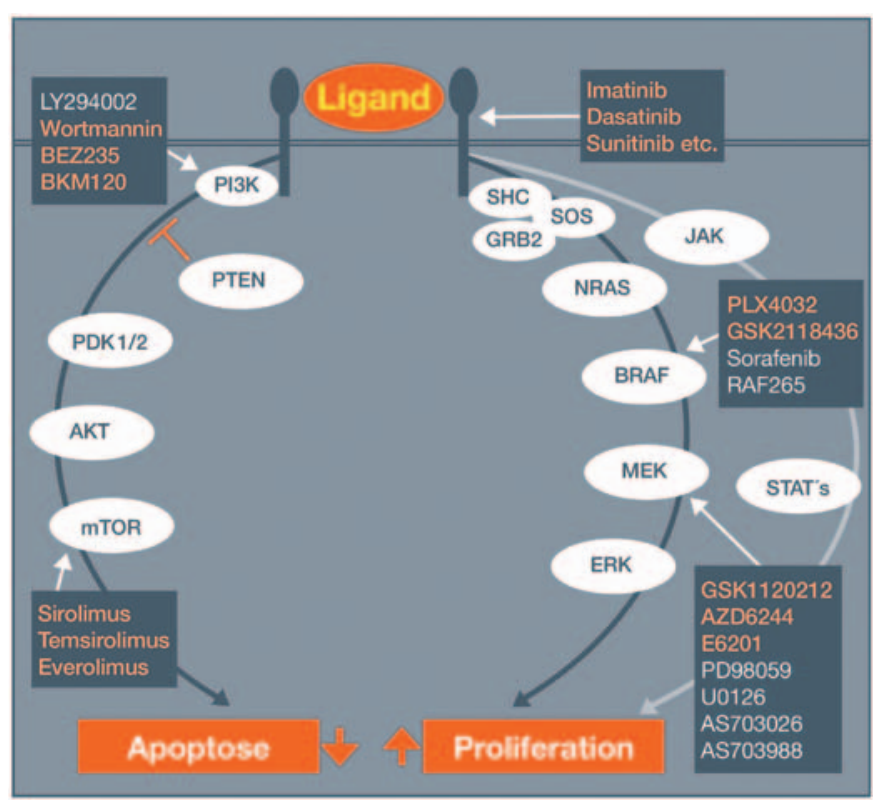

Abb. 3. Molekulare Targets beim malignen Melanom.

in der für die Phase-II-Studie empfohlenen Dosierung von $960 \mathrm{mg}$ bid behandelt wurden, betrug die objektive Ansprechrate $81 \%$. Von den 26 Respondern erreichten 24 eine PR und 2 eine CR. Auffallend war die schnelle Tumorregression und Besserung der Symptome innerhalb von 1-2 Wochen. Das mediane PFS wurde auf mehr als 7 Monate geschätzt, das mediane Gesamtüberleben konnte zum Zeitpunkt der Datenauswertung noch nicht beurteilt werden [15].

Unerwünschte Wirkungen des BRAF-Inhibitors manifestieren sich vor allem an der Haut und umfassen Exantheme, erhöhte Fotosensitivität, Juckreiz und palmoplantare Erythrodysästhesien. Besonders bemerkenswert sind die bei bis zu $30 \%$ der Patienten auftretenden papillomatösen, schnell proliferierenden, epithelialen Neoplasien, die wie Plattenepithelkarzinome imponieren.

Ein ähnliches Nutzen-Risiko-Profil wie PLX4032 zeigte in einer Phase-I/II-Studie auch der zweite derzeit in klinischen Studien untersuchte BRAF-Inhibitor, GSK2118436 [16].

Trotz der bei Patienten mit V600E-BRAF-Mutation beeindruckenden Ansprechraten ist davon auszugehen, dass BRAF-Inhibitoren als Monotherapie das Tumorwachstum nur vorübergehend unterdrücken können. Bemerkenswert ist dabei, dass auch Patienten mit objektivem Ansprechen (CR, PR) meist innerhalb relativ kurzer Zeit einen Rückfall erleiden. Dabei kann es sowohl zur Entwicklung neuer Metastasen (z.B. Hirnmetastasen) als auch zur Vergrößerung der bereits bestehenden Metastasen kommen.

MEK-Inhibitoren: Der oral applizierbare, selektive MEK1/2Inhibitor GSK1120212 wurde in einer 3-stufigen klinischen Studie bei Patienten mit unterschiedlichen soliden Tumoren und Lymphomen untersucht, darunter 29 Patienten mit meta- 
stasiertem Melanom. Von den 20 Patienten mit BRAF-Mutation-positivem Melanom, darunter 17 im Stadium M1c, sprachen $40 \%$ an (2 CR, 6 PR), bei den 22 Patienten mit BRAFWildtyp-Melanom wurden dagegen nur 2 PR berichtet [17].

\section{Zielstruktur c-KIT}

Bei bis zu 20\% der malignen Melanome sind Mutationen im c-KIT-Gen nachweisbar; besonders häufig finden sich die Genmutationen bei akralen und Schleimhautmelanomen (Tab. 1). Nachdem Einzelberichte eine gute Wirksamkeit von Imatinib beim c-KIT-positiven Melanom zeigten [18], wird die Wirksamkeit des oralen c-KIT-Inhibitors zurzeit in mehreren kontrollierten klinischen Studien untersucht.

\section{Fazit und Ausblick}

Selektive Inhibitoren der beim malignen Melanom mutierten Signaltransduktionswege, wie die beschriebenen BRAF-Inhibitoren, MEK-Inhibitoren und c-KIT-Inhibitoren, können bei Patienten mit Nachweis der entsprechenden Target-Mutation bemerkenswert schnell zu einer erheblichen Tumorregression und deutlichen Besserung subjektiv belastender Symptome führen. Bei weitgehendem Fehlen von Tumorlyse-Symptomen, aber häufig schweren kutanen Nebenwirkungen, darunter Plattenepithelkarzinom-ähnlichen Neoplasien, zeigen vor allem BRAF-Inhibitoren ein unerwartetes Nebenwirkungsprofil.

Trotz der hohen Aktivität dürften zielgerichtete Substanzen als Monotherapie allerdings keine Heilung des Melanoms ermöglichen. Eine Eradikation primär nicht sensitiver Tumorklone oder sekundär resistenter Tumorzellen und damit eine langfristige Rezidivprophylaxe im Sinne einer Chronifizierung der Erkrankung ist vermutlich nur möglich, wenn zusätzlich zu einer zielgerichteten Therapie noch andere Substanzen, insbesondere Immuntherapeutika, verabreicht werden. Valide Daten zu möglichen Kombinationen oder Sequenzen liegen bisher allerdings noch nicht vor.

\section{Disclosure Statement}

Die Autoren waren Referenten beim «1. Interdisziplinäres Expertenforum Hautkrebs», das von Bristol-Myers Squibb unterstützt wurde.

\section{Literatur}

1 Van Cutsem E, Lang I, D'haens G, et al.: KRAS status and efficacy in the first-line treatment of patients with metastatic colorectal cancer (mCRC) treated with FOLFIRI with or without cetuximab: The CRYSTAL experience. J Clin Oncol 2008;26 (suppl):abstr 2

2 Amado RG, Wolf M, Peeters M, et al.: Wild-type KRAS is required for panitumumab efficacy in patients with metastatic colorectal cancer. J Clin Oncol 2008;26:1626-1634.

3 Zhou C, Wu YL, Chen G, et al.: Efficacy results from the randomised phase III OPTIMAL (CTONG 0802) study comparing first-line erlotinib versus carboplatin (CBDCA) plus gemcitabine (GEM), in Chinese advanced non-small-cell lung cancer (NSCLC) patients (PTS) with EGFR activating mutations. ESMO 2010; abstr LBA13.

4 Mok TS, Wu YL, Thongprasert S, et al.: Gefitinib or carboplatin-paclitaxel in pulmonary adenocarcinoma. N Engl J Med 2009;361:947-957.

5 Yamamoto H, Toyooka S, Mitsudomi T: Impact of EGFR mutation analysis in non-small cell lung cancer. Lung Cancer 2009;63:315-321.

6 Blokx WAM, van Dijk MCRF, Ruiter DJ: Molecular cytogenetics of cutaneous melanocytic lesions diagnostic, prognostic and therapeutic aspects. Histopathology 2010;56:121-132.
7 Davies MA, Samuels Y: Analysis of the genome to personalize therapy for melanoma. Oncogene 2010;29:5545-5555.

8 Van de Vijver MJ, He YD, van't Veer LJ, et al.: A gene expression signature as a predictor of survival in breast cancer. N Engl J Med 2002;347:1999-2009.

$\checkmark$ Shepherd FA, Pereira JR, Ciuleanu T, et al.: Erlotinib in previously treated non-small-cell lung cancer. N Engl J Med 2005;353:123-132.

10 Bezjak A, Tu D, Seymour L, et al.: Symptom improvement in lung cancer patients treated with erlotinib: quality of life analysis of the National Cancer Institute of Canada Clinical Trials Group Study BR.21. J Clin Oncol 2006;24:3831-3837.

11 Lynch TJ, Bell DW, Sordella R, et al.: Activating mutations in the epidermal growth factor receptor underlying responsiveness of non-small-cell lung cancer to gefitinib. N Engl J Med 2004;350:21292139.

12 Fukuoka M, Wu Y, Thongprasert S, et al.: Biomarker analyses from a phase III, randomized, openlabel, first-line study of gefitinib versus carboplatin/ paclitaxel in clinically selected patients with advanced non-small cell lung cancer (NSCLC) in Asia (IPASS). J Clin Oncol 2009;27(suppl 15s): abstr 8006 .
13 Bang Y, Kwak EL, Shaw AT, et al.: Clinical activity of the oral ALK inhibitor PF-02341066 in ALKpositive patients with non-small cell lung cancer (NSCLC). J Clin Oncol 2010;28(suppl 18s):abstr 3, oral presentation.

14 Curtin JA, Fridlyand J, Kageshita T, et al.: Distinct sets of genetic alterations in melanoma. $\mathrm{N}$ Engl $\mathrm{J}$ Med 2005:353:2135-2147.

15 Flaherty KT, Puzanov I, Kim KB, et al.: Inhibition of mutated, activated BRAF in metastatic melanoma. N Engl J Med 2010;363:809-819.

16 Kefford R, Arkenau H, Brown MP, et al.: Phase I/II study of GSK2118436, a selective inhibitor of oncogenic mutant BRAF kinase, in patients with metastatic melanoma and solid tumors. J Clin Oncol 2010;28(suppl 15s):abstr 8503, oral presentation.

17 Infante JR, Fecher LA, Nallapareddy S, et al.: Safety and efficacy results from the first-in-human study of the oral MEK 1/2 inhibitor GSK1120212. J Clin Oncol 2010;28(suppl 15s):abstr 2503, oral presentation.

18 Hodi FS, Friedlander P, Corless CL, et al.: Major response to imatinib mesylate in KIT-mutated melanoma. J Clin Oncol 2008;26:2046-2050. 